\title{
FATORES AMBIENTES E INICIAÇ̃̃O FLORAQL DE CANA-DE-AÇÚCAR, NO ANO AGRÍCOLA 1984/85, NA REGIÃO DE PIRACICABA, SP (')
}

\author{
MARISA VAZQUEZ CARLUCCI $(2,5)$, \\ NEUSA DINIZ DA CRUZ $\left({ }^{3}\right)$ e RAFAEL ALVAREZ $\left({ }^{4}\right)$
}

\section{RESUMO}

Com o objetivo de determinar as épocas de iniciação e emergência da inflorescência da cana-de-açúcar (Saccharum spp.), correlacionando-as aos fatores climáticos, coletaram-se amostras dos cultivares NA 56-79, SP 70-1143 e IAC 52-150, em experimentos na região de Piracicaba, SP. De cada cultivar, colheram-se, semanalmente, dez palmitos dos colmos mais vigorosos, de 20 de fevereiro a 3 de julho de 1984. Analisaram-se a diferenciação e o desenvolvimento floral através de cortes histológicos da regiāo apical e de medidas do comprimento da inflorescência. Coletaram-se os dados climáticos, durante o período de indução (25 de fevereiro-20 de março) no posto meteorológico da Estação Experimental do Instituto Agronômico, em Piracicaba. A ocorrência de temperaturas elevadas (Tmáx> $\left.31^{\circ} \mathrm{C}\right)$, a baixa precipitação $(47,3 \mathrm{~mm})$ e a alta freqüência de dias $(76 \%) \mathrm{com}$ diferença entre temperaturas máximas e mínimas acima de $13^{\circ} \mathrm{C}$ no período indutivo, afetaram a diferenciação floral. Os cultivares NA 56-79 e SP 70-1143 não floresceram e o 'IAC 52-150' apresentou uma redução de cerca de $50 \%$ no florescimento. O primeiro estádio reconhecivel da iniciação floral foi detectado em 6 de abril, quando o domo apical media $0,011 \mathrm{~cm}$, só ocorrendo a emergência da panícula na primeira semana de julho.

Termos de indexação: cana-de-açúcar, Saccharum spp., iniciaçāo floral, fatores climáticos.

(1) Recebido para publicação em 28 de setembro de 1987 e aceito em 14 de março de 1988.

(2) Atualmente na Seção de Floricultura e Plantas Ornamentais, Instituto Agronômico (IAC), Caixa Postạl 28, 13001 Campinas (SP).

( $\left.{ }^{3}\right)$ Seção de Citologia, IAC,

(4) Seção de Cana-ce-Açúcar, IAC.

(5) Com boisa de pesquisa do CNPq. 


\section{INTRODUÇÃO}

Para a maioria dos cultivares de cana-de-açúcar, a indução floral ocorre com fotoperíodo de doze a doze e meia horas (COLEMAN, 1960; ARCENEAUX, 1967; CLEMENTS \& AWADA, 1967). Há necessidade, no entanto, de outras condições climáticas específicas, durante e após a indução, para a iniciação e desenvolvimento da inflorescência.

Temperaturas mínimas abaixo de $18^{\circ} \mathrm{C}$ (COLEMAN, 1963; PALIATSEAS, 1963; PEREIRA et al., 1983, 1986) e máximas acima de $31^{\circ} \mathrm{C}$ (ELLIS et al., 1967; JULIEN et al., 1974), assim como deficiência hídrica, no periodo indutivo, levam, em geral, a um atraso na iniciação e desenvolvimento das panículas, bem como diminuem o número de panículas formadas (COLEMAN, 1960; CHU \& SERAPION, 1971; HUMBERT et al., 1969).

PANJE \& SRINIVASAN (1960) constataram um atraso de catorze dias na floração da maioria dos clones estudados de $S$. spontaneum quando, no período indutivo, a precipitação foi de $74 \mathrm{~mm}$ apenas. PEREIRA et al. (1986), analisando dados climáticos durante a indução, de 1972 a 1985, na região de Araras, SP, constataram, em relação ao cultivar NA 56-79, que nos anos com florescimento a precipitação média foi de $198 \mathrm{~mm}$ em dez dias, contra $65 \mathrm{~mm}$ em seis dias nos anos em que o florescimento não ocorreu.

YEU (1980) e ELLIS et al. (1967) salientam que a menor diferença entre as temperaturas extremas, a maior precipitação e o maior número de dias com chuva no período indutivo, favorecem uma floração intensa. Ainda em relação à diferença entre temperaturas extremas, PEREIRA et al. (1986) observaram que, em anos com florescimento, a diferença média entre temperatura máxima e mínima tem sido de aproximadamente $10^{\circ} \mathrm{C}$ contra $14^{\circ} \mathrm{C}$ nos anos em que as plantas não floresceram.

Outro fator importante relacionado à temperatura, é a freqüência de dias e/ou noites com temperaturas não indutivas. PALIATSEAS (1963) mostra haver um efeito cumulativo da temperatura. Para COLEMAN (1963), aparentemente $50 \%$ de noites com temperatura igual ou menor que $18^{\circ} \mathrm{C}$ no período indutivo inibe a indução floral.

Sabe-se que diferentes cultivares se comportam diferentemente em relação ao número de dias necessários, dentro do período indutivo, para a formação do estímulo floral (COLEMAN, 1969). A quantidade necessária desse estímulo pode levar à diferenciação da panícula no início ou no final do período indutivo.

Alguns métodos têm sido testados para estimar a data de início de acúmulo do estímulo floral. Um deles, utilizado por CLEMENTS (1975), consiste no acompanhamento, ao microscópio, de alteraçōes anatômicas do meristema apical. Trabalhando com a variedade NCO 310 , esse autor determinou que o pri- 
meiro estádio reconhecivel da formação da inflorescência foi detectado quando o domo apical media cerca de $0,010 \mathrm{~cm}$, e supōe que o estímulo fotoperiódico deva ter-se acumulado no ápice dez a catorze dias antes da iniciação floral.

$O$ objetivo do presente trabalho foi determinar, na região canavieira de Piracicaba, SP, a época de iniciação floral de alguns cultivares de cana-de-açúcar, correlacionando o processo às condições climáticas do periodo indutivo.

\section{MATERIAL E MÉTODOS}

O experimento foi instalado na Usina Modelo, em Piracicaba, SP, utilizando-se os cultivares NA 56-79, SP 70-1143 e IAC 52-150, que, normalmente, florescem na regiāo. As plantas estavam no estádio de soqueira, realizando-se o corte no final de agosto de 1983. O espaçamento entre as linhas era de $1,40 \mathrm{~m}$.

A partir de $20 / 2$ até $3 / 7$ de 1984 , coletaram-se semanalmente dez palmitos dos colmos mais vigorosos de cada cultivar. A regiāo apical foi removida e fixada em FAA $50 \%$, desidratada na série de álcool butílico terciário e embebida em parafina. Em seguida, o material foi seccionado em cortes longitudinais seriados de $10 \mu \mathrm{m}$, sendo os melhores fotomicrografados. O tamanho da inflorescência foi medido desde a diferenciação floral até o início da abertura do cartucho.

Dados de temperaturas mínimas (Tmín) e máximas (Tmáx), assim como o número de dias de chuva e precipitação pluvial total, coletados durante o período fotoindutivo, no posto meteorológico da Estação Experimental do Instituto Agronômico, em Piracicaba, encontram-se no quadro 1. O período fotoindutivo nessa região $\left(22^{\circ} 43^{\prime} \mathrm{S}\right.$.) ocorre entre $25 / 2$ e $20 / 3$, conforme demonstrado por BARBIERI et al. (1981).

A avaliação final da porcentagem de florescimento foi realizada em 27 de agosto, dissecando-se os ápices de 300 colmos de cada cultivar. Depois de examinar o ponto de crescimento apical, anotou-se a freqüência de colmos sem indução, induzidos, encartuchados e florescidos: foram considerados induzidos colmos com panículas de até $10 \mathrm{~cm}$. Acima desse tamanho, eles foram classificados como encartuchados ou florescidos.

\section{RESULTADOS E DISCUSSÃO}

Através dos dados do quadro 1, pode-se observar que as condições de temperatura e precipitação, em 1984, durante o período indutivo, na região de $\mathrm{Pi}$ racicaba, não foram favoráveis ao florescimento de cana-de-açúcar. Isso ficou demonstrado através da avaliação da porcentagem do florescimento, realizada em 27 de agosto, quando se observou que os cultivares NA 56-79 e SP 70-1143 
não apresentaram iniciação floral e que o 'IAC 52-150', de florescimento freqüente na região da cana-de-açúcar, expressou-a na seguinte proporção:

Colmos florescidos e encartuchados . . . . . . . . . 28,4\%;

Colmos induzidos . . . . . . . . . . . . . . . . 21,2\%;

Colmos não induzidos $\ldots \ldots \ldots \ldots \ldots \ldots \ldots \ldots \ldots \ldots .4 \ldots \ldots$.

QUADRO 1. Temperatura máxima, mínima, amplitude térmica e precipitação diárias obtidas no posto meteorológico da Estação Experimental de Piracicaba (SP) em 25 de fevereiro-20 de março de 1984. Fonte dos dados: Seção de Climatologia Agrícola

\begin{tabular}{|c|c|c|c|c|c|}
\hline Mês & Dia & $\begin{array}{l}\text { Temperatura } \\
\text { máxima }\end{array}$ & $\begin{array}{l}\text { Temperatura } \\
\text { mínima }\end{array}$ & $\begin{array}{l}\text { Amplitude } \\
\text { térmica }\end{array}$ & Chuva \\
\hline & & ${ }^{\circ} \mathrm{C}$ & ${ }^{\circ} \mathrm{C}$ & & $\mathrm{mm}$ \\
\hline \multirow[t]{5}{*}{ Fev. } & 25 & 35,0 & 18,9 & 16,1 & - \\
\hline & 26 & $34,3^{\circ}$ & 20,0 & 14,3 & - \\
\hline & 27 & 36,0 & 18,9 & 17,1 & - \\
\hline & 28 & 35,4 & 21,1 & 14,3 & 3,0 \\
\hline & 29 & 36,0 & 21,6 & 14,4 & - \\
\hline \multirow[t]{20}{*}{ Março } & 01 & 34,3 & 20,6 & 13,7 & - \\
\hline & 02 & 35,4 & 21,2 & 14,2 & - \\
\hline & 03 & 34,9 & 19,5 & 15,4 & - \\
\hline & 04 & 34,0 & 19,3 & 14,7 & - \\
\hline & 05 & 33,9 & 20,9 & 13,0 & 8,1 \\
\hline & 06 & 29,9 & 21,2 & 8,7 & 7,4 \\
\hline & 07 & 28,8 & 20,2 & 8,6 & 6,3 \\
\hline & 08 & 30,4 & 20,5 & 9,9 & 22,5 \\
\hline & 09 & 31,2 & 19,2 & 12,0 & - \\
\hline & 10 & 32,9 & 19,9 & 13,0 & - \\
\hline & 11 & 32,6 & 21,6 & 11,0 & - \\
\hline & 12 & 33,4 & 18,3 & 15,1 & - \\
\hline & 13 & 33,8 & 18,4 & 15,4 & - \\
\hline & 14 & 31,6 & 20,2 & 11,4 & - \\
\hline & 15 & 32,4 & 18,6 & 13,8 & - \\
\hline & 16 & 32,8 & 17,0 & 15,8 & - \\
\hline & 17 & 32,7 & 18,0 & 14,7 & - \\
\hline & 18 & 35,4 & 16,7 & 18,7 & - \\
\hline & 19 & 33,2 & 17,2 & 16,0 & - \\
\hline & 20 & 34,5 & 17,3 & 17,2 & - \\
\hline
\end{tabular}


Pelo quadro 1, salienta-se que, apesar de, no periodo indutivo, ter ocorrido elevado número de noites (isto é, 21) com Tmín $\geqslant 18^{\circ} \mathrm{C}$, o número de dias com Tmáx $\angle 31^{\circ} \mathrm{C}$ foi muito reduzido (isto é, 3), e em somente três dias durante o período de indução, a Tmáx e a Tmin estiveram dentro dos limites ideais de $31^{\circ} \mathrm{C}$ (ELLIS et al., 1967; JULIEN et al., 1974) e $18^{\circ}$ (COLEMAN, 1963; PALIATSEAS, 1963; PEREIRA et al., 1986) respectivamente.

Ainda com relação às temperaturas, pode-se notar que ocorreram 12 dias consecutivos com Tmáx $>31^{\circ} \mathrm{C}$. A diferença média entre Tmáx e Tmín foi aproximadamente $14^{\circ} \mathrm{C}$ e apenas em seis dias (isto é, 24\%), essas diferenças foram menores que $13^{\circ} \mathrm{C}$. $O$ efeito cumulativo de temperaturas não indutivas, reduzindo ou inibindo a floração, foi mencionado por PALIATSEAS (1963) e COLEMAN (1963). PEREIRA et al. (1986) encontraram nos anos com florescimento uma diferença média Tmáx-Tmín, no periodo indutivo, de cerca de $10^{\circ} \mathrm{C} \mathrm{e}$, nos anos sem florescimento, de $14^{\circ} \mathrm{C}$, o que concorda com os nossos resultados. Esses autores observaram ainda que anos floriferos tiveram $82 \%$ dos dias com diferenças menores que $13^{\circ} \mathrm{C}$ contra $60 \%$ naqueles sem florescimento, situação essa até menos prejudicial do que a ocorrida em 1984, na região de Piracicaba.

A quantidade e os dias de chuva também foram fatores com efeito negativo na formação da panícula. A precipitação total foi somente de $47,3 \mathrm{~mm}$, concentrada basicamente entre os dias 5 e 8 de março. CHU \& SERAPION (1971) e PANJE \& SRINIVASAN (1960) também observaram que a deficiência hídrica atrasa o florescimento, e HUMBERT et al. (1969) encontraram redução no número de panículas formadas sob estresse de água. Em resultados semelhantes aos nossos, obtidos por PEREIRA et al. (1986) em anos sem florescimento, a precipitação total foi de $65 \mathrm{~mm}$ somente em seis dias.

Uma vez que maior precipitação e maior número de dias com chuva diminuem a ocorrência de temperaturas elevadas e amenizam a diferença entre as temperaturas extremas (ELLIS et al., 1967; YEU, 1980), fica dificil separar o efeito desses dois fatores em condições de campo.

Analisando microscopicamente os ápices dos cultivares IAC 52-150, NA 56-79 e SP 70-1143, colhidos durante e após o período indutivo, foi possivel acompanhar as modificações ocorridas desde o estádio vegetativo até o da inflorescência jovem, para o cultivar IAC 52-150 (Figura 1). Os cultivares NA 56-79 e SP 70-1143 permaneceram em estádio vegetativo.

A figura $1 \mathrm{~A}$ mostra o ápice em estádio vegetativo representativo da coleta de 30 de março de 1984, e a figura 1B (coleta de 6 de abril), um alongamento do domo apical marcando a mudança do estádio vegetativo para o reprodutivo: nessa ocasião, o domo apical media cerca de $0,011 \mathrm{~cm}$, semelhante ao tamanho e à forma descrita por CLEMENTS (1975) para essa fase. 

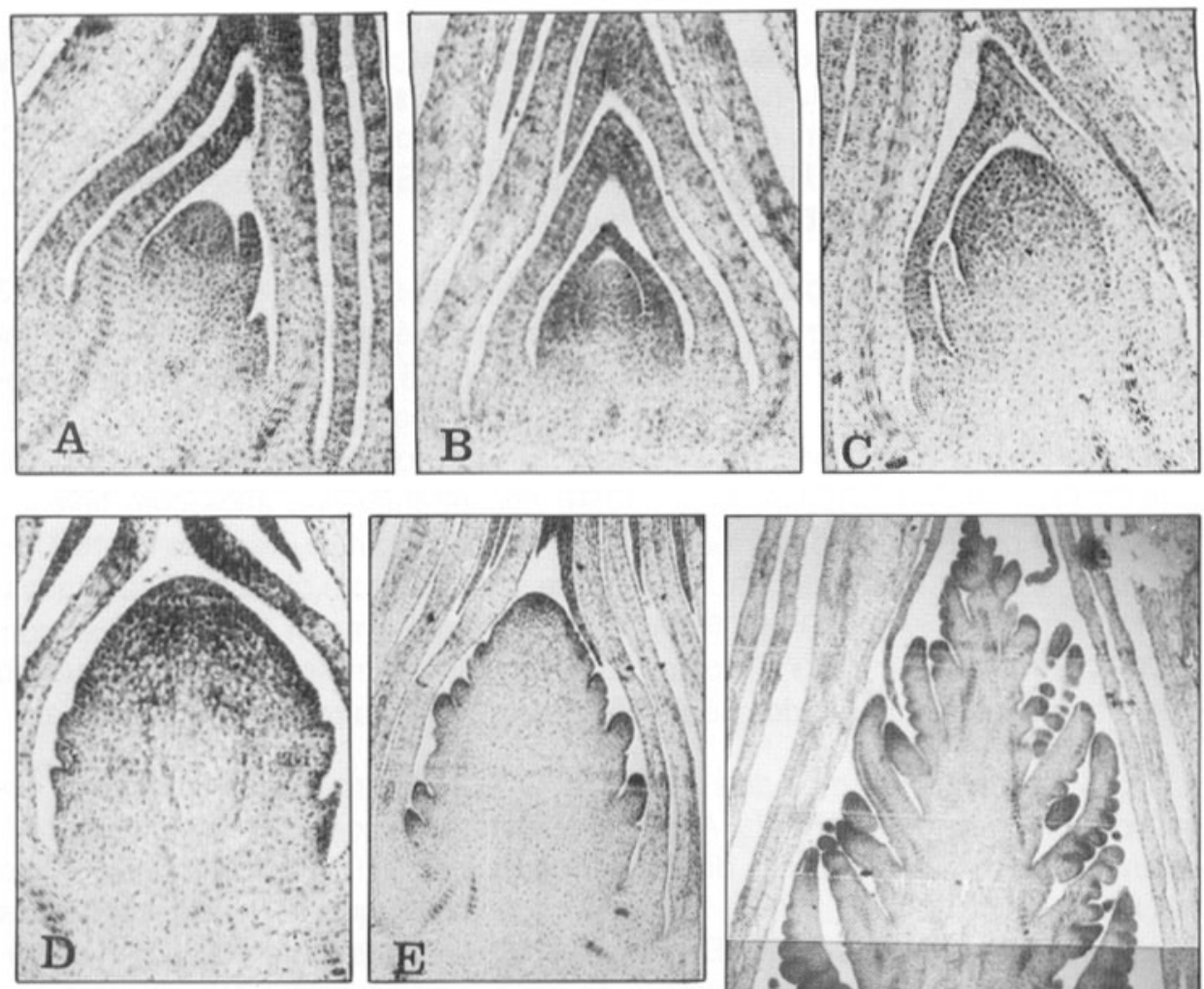

FIGURA 1. Estádios de desenvolvimento do ápice em cana-de-açúcar cultivar IAC 52-150: A: ápice vegetativo; B: ápice em transiçāo do estádio vegetativo para o reprodutivo; C: primeiro estádio reconhecível do primórdio de inflorescência; D: primórdio de inflorescência mais

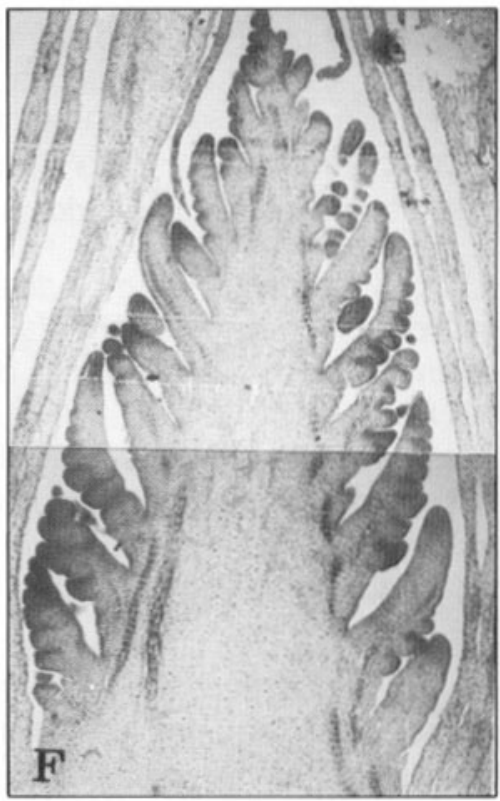
desenvolvido; E: primórdio de inflorescência com início de ramificação, e F: inflorescência jovem com ramificações primárias e secundárias. (Figuras 1A, 1B, 1C, 1D, 1E e 1F, respectivamente, 100x, 82x, 56x, 60x, $23 x$ e $20 x$ )

Era de esperar, entretanto, que a iniciação ocorresse em meados de março, em resposta ao fotoperíodo indutivo: supōe-se, porém, que, devido às condições de estresse hídrico e das altas temperaturas, a metabolização e a 
translocação do estímulo floral ficaram prejudicadas, atrasando, portanto, a iniciação do primórdio da inflorescência em cerca de vinte dias.

Efeito de condiçōes climáticas extremas sobre o metabolismo do estímulo floral foi também mencionado por COLEMAN (1969).

Nas figuras 1C, 1D e 1E, representativas das coletas efetuadas em 13, 19 e 24 de abril de 1984 respectivamente, observa-se considerável aumento no tamanho do primórdio da inflorescência, com a formação concomitante de ramificaçōes primárias da panícula.

A figura 1F mostra uma inflorescência jovem com ramificações primárias e secundárias de ápices colhidos em 11 de maio, medindo em torno de $0,4 \mathrm{~cm}$ de comprimento.

O tamanho da inflorescência nos estádios subseqüentes, de coletas de 18 de maio a 3 de julho, está representado na figura 2.

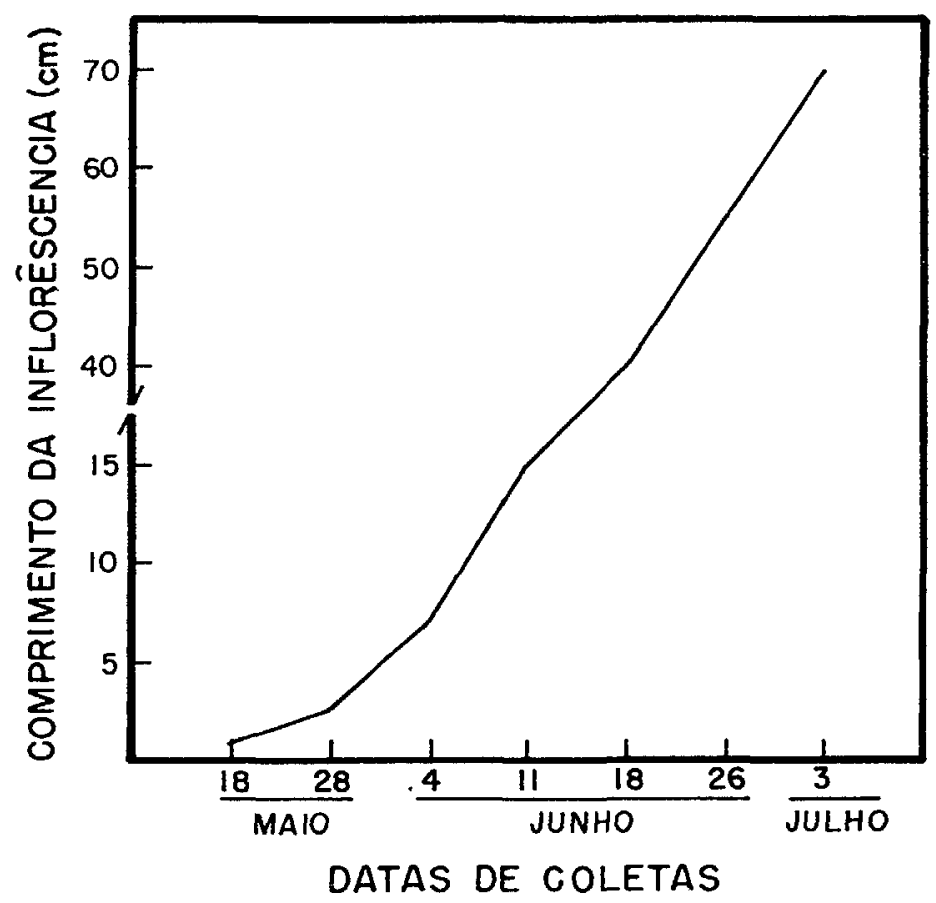

FIGURA 2. Desenvolvimento da inflorescência de cana-de-açúcar, cultivar IAC 52-150, no periodo 18 de maio-3 de julho de 1984. 
Para o cultivar IAC 52-150, na região de Piracicaba, as primeiras inflorescências emergem em fins de maio/início de junho. Entretanto, sob as condiçōes de seca que ocorreram de fevereiro a julho de 1984, essa emergência só se verificou na primeira semana de julho, com atraso de trinta dias, portanto.

\section{CONCLUSÕES}

1. As condições climáticas desfavoráveis, principalmente altas temperaturas e deficiência hídrica, durante o periodo indutivo, influíram negativamente no florescimento dos cultivares NA 56-79, SP 70-1143 e IAC 52-150.

2. Os cultivares estudados apresentaram-se com diferentes sensibilidades aos fatores ambientes que interferiram no florescimento. O 'NA 56-79' e 'SP 70-1143' mostraram-se mais sensiveis e não floresceram, enquanto o 'IAC 52-150', menos exigente, floresceu 50\%.

3. Os periodos de iniciação e de emergência floral no 'IAC 52-150' foram atrasados de vinte a trinta dias, respectivamente, em relação às datas esperadas - meados de março, para a iniciação floral, e início de junho, para a emergência da panícula.

\section{SUMMARY}

ENVIRONMENTAL EFFECTS ON FLORAL INITIATION OF SUGARCANE DURING THE 84/85 SEASON, IN THE PIRACICABA REGION, STATE OF SÃO PAULO, BRAZIL

In order to determine the date of initiation and emergence of sugarcane inflorescences, samples from cultivars NA 56-79, SP 70-1143 and IAC 52-150 were collected from field trials conducted in Piracicaba region. Shoot apices from 10 vigorous stalks, from each cultivar, were collected once each week from February 20th to July 3rd, 1984. Floral differentiation and development were analysed by microscopic examination of median longitudinal sections of shoot apices, as well as measurements of length of inflorescences. During the critical period of induction - from February 25th to March 20th - all climatic data were collected at the Piracicaba Experimental Station of the Instituto Agronômico in the same climatological area. Temperatures higher than $31^{\circ} \mathrm{C}$, low rainfall, $47,3 \mathrm{~mm}$, and the hight percentage $(76 \%)$ of days with more than $13^{\circ} \mathrm{C}$ difference between maximum and minimum temperatures during the critical period of induction, affected the floral differentiation. The cultivars NA 56-59 and SP 70-1143 did not flower while the cultivar IAC 52-50 flowered only $50 \%$. The earlier recognizable inflorescence primordia was detected on April 6th, when the dometype structure measured $0.011 \mathrm{~cm}$, and the emergence panicle occurred during the first week of July.

Index terms. : sugarcane, Saccharum spp., floral initiation, climatic factors. 


\section{REFERÊNCIAS BIBLIOGRÁFICAS}

ARCENEAUX, G. Flowering of sugarcane. In: CONGRESS OF THE INTERNATIONAL SOCIETY OF SUGAR CANE TECHNOLOGISTS, 12., San Juan, 1965. Proceedings. Amsterdam, Elsevier, 1967. p.780-784.

BARBIERI, V.; BACCHI, O.O.S.; BASSINELO, A.I. \& VILLA NOVA, N.A. Estudos dos efeitos do clima no florescimento da cana-de-açúcar. In: CONGRESSO NACIONAL DA SOCIEDADE DOS TÉCNICOS AÇUCAREIROS E ALCOOLEIROS DO BRASIL, 2., Rio de Janeiro, 1981. Anais. v.3, p.341-362.

CHU, T.L. \& SERAPION, J.L. Flower initiation and tassel, emergence in sugar. cane. Journal Agricultural University of Puerto Rico, Rio Pedras, 55:101-115, 1971.

CLEMENTS, H.F. Flowering of sugarcane: mechanics and control. Honolulu, University of Hawaii, 1975. 56p. (Technical Bulletin, 92)

\& AWADA, M. Experiments on the artificial induction of flowering in sugarcane. In: CONGRESS OF THE INTERNATIONAL SOCIETY OF SUGAR CANE TECHNOLOGISTS, 12., San Juan, 1965. Proceedings. Amsterdam, Elsevier, 1967. p.795-812.

COLEMAN, E.R. Effect of temperature on flowering in sugar cane. International Sugar Journal, 6:351-353, 1963.

. Factors involved in the flowering of sugarcane (Saccharum spp.). In: CONGRESS OF THE INTERNATIONAL SOCIETY OF SUGAR CANE TECHNOLOGISTS, 10., Hawaii, 1959. Proceedings. Amsterdam, Elsevier, 1960. p.805-814.

. Physiology of flowering in sugarcane. In: CONGRESS OF THE INTERNATIONAL SOCIETY OF SUGAR CANE TECHNOLOGISTS, 13., Taiwan, 1968. Proceedings. Amsterdam, Elsevier, 1969. p.992-1000.

ELLIS, T.O.; BREEMEN, J.F. van \& ARCENEAUX, G. Flowering of sugarcane in relation to maximum temperature during the induction period. In: CONGRESS OF THE INTERNATIONAL SOCIETY OF SUGAR CANE TECHNOLOGISTS, 12., San Juan, 1965. Proceedings. Amsterdam, Elsevier, 1967. p.790-794.

HUMBERT, R.P.; LIMA, G.M. \& GOVEAS, J. Tassel control progress with reglone in the mexican sugar industry. In: CONGRESS OF THE INTERNATIONAL SOCIETY OF SUGAR CANE TECHNOLOGISTS, 13., Taiwan, 1968. Proceedings. Amsterdam, Elsevier, 1969. p.462-467.

JULIEN, R.; SOOPRAMANIEN, G.C. \& LORENCE, D. Juvenility, senility, climate and flowering in Saccharum. In: CONGRESS OF THE INTERNATIONAL SOCIETY OF SUGAR CANE TECHNOLOGISTS, 15., Durban, 1974. Proceedings: Durban. Hayne \& Gibson, 1974. p.984-990.

PALIATSEAS, E.D. Further studies on flowering of sugar cane in Louisiana. In: CONGRESS OF THE INTERNATIONAL SOCIETY OF SUGAR CANE TECHNOLOGISTS, 11., Mauritius, 1962. Proceedings: Amsterdam, Elsevier, 1963. p.805-814.

PANJE, R.R. \& SRINIVASAN, K. Studies in Saccharum spontaneum. A note on the flowering sequence of Saccharum spontaneurn clones. In: CONGRESS OF THE INTERNATIONAL SOCIETY OF SUGAR CANE TECHNOLOGISTS, 10., Hawaii, 1959. Proceedings. Amsterdam, Elsevier, 1960. p.819-824. 
PEREIRA, A.R.; BARBIERI, V. \& MANIERO, M.A. Condicionamento climático da indução ao florescimento em cana-de-açúcar. Revista da Sociedade dos Técnicos Açucareiros e Alcooleiros do Brasil, Piracicaba 4(6):56-59, 1986.

\& VILLA NOVA, N.A. Condicionamento climático da induçāo ao florescimento em cana-de-açúcar. Boletim Técnico PLANALSUCAR, Piracicaba, 5(3):5-14, 1983.

YEU, W.K. Studies on flowering of sugar cane in the South of Haiwan, China. In: CONGRESS OF THE INTERNATIONAL SOCIETY OF SUGAR CANE TECHNOLOGISTS, 17, Manila, 1980. Proceedings. Makati, Metro Manila, 1980. p.1301-1306. 